\title{
BANACH ALGEBRAS WHICH ARE A DIRECT SUM OF DIVISION ALGEBRAS
}

\author{
ANTONIO FERNANDEZ LOPEZ and EULALIA GARCIA RUS
}

(Received 2 December 1986)

Communicated by J. F. Price

\begin{abstract}
In this note it is proved that a (real or complex) semiprime Banach algebra $A$ satisfying $x A x=x^{2} A x^{2}$ for every $x \in A$ is a direct sum of a finite number of division Banach algebras.

1980 Mathematics subject classification (Amer. Math. Soc.): primary 16 A 48; secondary 46 H 20.

Keywords and phrases: Banach algebra, semiprime, division algebra.
\end{abstract}

\section{Introduction}

Recently Esterle and Oudadess (1986) have shown that a complex semisimple Banach algebra $A$ satisfying $A x^{2}=A x$ for every $x \in A$ is isomorphic to $\mathbb{C}^{n}$ for some $n \geq 0$. Their strategy consists of proving that such an algebra is commutative and then to obtain the result by using standard techniques of spectral theory. These methods do not extend to the case of real scalars since there exist noncommutative real Banach algebras $B$ (for instance, the quaternions) which are semisimple and satisfy $B x^{2}=B x$ for every $x \in B$.

We prove in this note that a (real or complex) semiprime Banach algebra $A$ satisfying $x^{2} A x^{2}=x A x$ for every $x \in A$ is a direct sum of a finite number of ideals each of which is isomorphic to either the reals, complexes or quaternions. If $A$ is complex all of them are isomorphic to the complex field and $A$ is commutative. In particular, the result of Esterle and Oudadess can be derived from our theorem. We are indebted to our colleague Professor J. A. Cuenca who pointed

(C) 1988 Australian Mathematical Society $0263-6115 / 88 \$ A 2.00+0.00$ 
out to us that the hypothesis on the existence of a unit element was superfluous in the first version of our result.

\section{The theorem}

Let $A$ be an (associative) algebra over a field $F$. The algebra $A$ is called semiprime if $I^{2}=0$ implies $I=0, I$ right ideal of $A$ (equivalently, $x A x=0$ implies $x=0$ ). For a semiprime algebra $A$ the socle is defined to be the sum of all minimal right ideals of $A$ (= sum of all minimal left ideals). An associative algebra $A$ is said to be unital if there exists $1 \in A$ such that $1 x=x 1=x$ for every $x \in A$. A unital algebra $A$ is called a division algebra if every non-zero element in $A$ is invertible. A nonzero idempotent $e \in A$ is called primitive, completely primitive or principal respectively, if $e A e$ has no idempotents other than $0, e, e A e$ is a division algebra or $(1-e) A(1-e)$ does not contain any nonzero idempotent respectively, where $(1-e) A(1-e)$ makes sense even if $A$ is non-unital. An element $a \in A$ is von Neumann regular if we can find $b \in A$ such that $a=a b a$.

THEOREM. Every (real or complex) semiprime Banach algebra $A$ satisfying $x A x=x^{2} A x^{2}$ for every $x \in A$ is a direct sum $A=M_{1} \oplus \cdots \oplus M_{n}$ of ideals each of which is isomorphic to either the reals, complexes or quaternions. If $A$ is complex all the $M_{i}$ are isomoprhic to the complex field and $A$ is commutative. Conversely, every direct sum $A=M_{1} \oplus \cdots \oplus M_{n}$ of division Banach algebras is a semisimple Banach algebra satisfying $x A x=x^{2} A x^{2}$.

ProOF. Since the reverse implication is clear we only must settle the necessary condition. Without loss in generality we may assume $A \neq 0$. First of all we note that $x A x=x^{n} A x^{n}$ for all $x \in A, n \geq 1$. Now it follows by semiprimeness that $A$ has no nilpotent elements. Hence for any non-zero $x \in A, x^{3} \neq 0$, and since $x^{3} \in x A x=x^{3} A x^{3}$ there is $y \in A$ such that $x^{3}=x^{3} y x^{3}$, so that $x^{3} y$ and $y x^{3}$ are non-zero idempotents. Suppose that $\left\{e_{n}\right\}_{n=1}$ is an infinite sequence of non-zero orthogonal idempotents and let $\left\{\lambda_{n}\right\}_{n=1}$ be a sequence of non-zero real numbers such that $\sum\left\|\lambda_{n} e_{n}\right\|<\infty$. If $u=\sum \lambda_{n} e_{n}$ we have $u \neq 0$ and $u^{3}=\sum \lambda_{n}^{3} e_{n}$ is a von Neumann regular element, so that we can use the arguments of Kaplansky (1948), Lemma 1, to get a contradiction. Thus $A$ contains a principal idempotent $\nu$ which is a sum $\nu=e_{1}+\cdots+e_{r}$ of orthogonal primitive idempotents; we show that $\nu$ is a unit element of $A$ and that every primitive idempotent $e \in A$ is completely primitive. Indeed, $B=(1-\nu) A(-\nu)$, is a Banach algebra satisfying the same conditions as $A$, so that $B=0$ since otherwise $B$ would contain a non-zero idempotent, which is a contradiction because $\nu$ is principal. Then $((1-\nu) A)^{2}=0$ and hence $(1-\nu) A=0$ by semiprimeness. 
Analogously $A(1-\nu)=0$, so that $\nu$ is a unit element of $A$. Now let $e$ be a primitive idempotent of $A$. Since the algebra $e A e$ satisfies the same conditions as $A$ we have that for every non-zero element $x \in e A e$ there exists $y \in e A e$ such that $x^{3} y$ and $y x^{3}$ are non-zero idempotents in $e A e$. Hence $x^{3} y=e=y x^{3}$ since $e$ is primitive. Then $e A e$ is a divison algebra and therefore $e$ is a completely primitive idempotent. We have thus proved that $A$ is a unital algebra coinciding with its socle (Jacobson (1956), page 65). But such an algebra is semisimple and satisfies the Descending Chain Condition on right ideals (Jacobson (1956), page 75), so that by the Wedderburn-Artin theorem (Jacobson (1956), page 40) $A=M_{1} \oplus \cdots \oplus M_{n}$ is a direct sum of ideals each of which is isomorphic to a full matrix ring $M_{n(i)}\left(D_{i}\right)$ over a division ring $D_{i}$. Since $A$ contains no nilpotent elements, every $n_{i}=1$ and since every $D_{i}$ is a division Banach algebra (in fact, $D_{i} \cong u_{i} A u_{i}$ for a completely primitive idempotent $u_{i}$; (Jacobson (1956), Lemma 3.1)), we have by Mazur-Gelfand theorem (Rickart (1974), Theorems 1.7.1 and 1.7.6) that each $D_{i}$ is isomorphic to either the reals, complexes or quaternions. If $A$ is complex then every $D_{i}$ is isomoprhic to the complex field and $A$ is commutative, which completes the proof.

We finish by noting that the theorem of Esterle and Oudadess can be derived from our result. Indeed, let $A$ be a complex semiprime Banach algebra satisfying $A x=A x^{2}$ for every $x \in A$. Since $A$ is commutative (Esterle and Oudadess (1986), Lemma 3.1) $x A x=x^{2} A x^{2}$ for every $x \in A$, so that we can apply our theorem to conclude that $A \cong \mathbb{C}^{n}$ for some $n \geq 0$.

\section{References}

J. Esterle and M. Oudadess (1986), 'Structure of Banach algebras $A$ satisfying $A x^{2}=A x$ for every $x \in A^{\prime}$, Proc. Amer. Math. Soc. (1) 96, 91-94.

N. Jacobson (1956), Structure of rings, (Amer. Math. Soc. Colloq. Publ., vol. 37, Amer. Math. Soc., Providence, R.I.).

I. Kaplansky (1948), 'Regular Banach algebras', J. Indian Math. Soc. 12, 57-62.

C. E. Rickart (1974), General theory of Banach algebras, (Krieger).

Departamento de Algebra,

Geometria y Topologia

Universidad de Malaga

29080 Malaga

Spain 